\title{
Comparisons of Electron Temperature and Density, and Ion Temperature Profiles in the TJ-II Stellarator
}

\author{
Josep Maria FONTDECABA, Ignacio PASTOR, Juan ARÉVALO, Jesús HERRANZ, \\ Kieran J. McCARTHY and Guillermo SÁNCHEZ BURILLO \\ Laboratorio Nacional de Fusión, Asociación EURATOM-Ciemat, 28040 Madrid Spain
}

(Received 7 December 2009 / Accepted 24 March 2010)

\begin{abstract}
Charge exchange-neutral particle analyzer diagnostics are used to obtain majority ion temperature profiles for plasmas created in the TJ-II stellarator. However, because of technical limitations, measurements are limited to two radial positions per discharge. Therefore plasma reproducibility for a large number of discharges is essential for performing scans across the plasma minor radius and hence for obtaining ion temperature profiles. Such conditions have recently been achieved in the TJ-II by the implementation of a lithium wall coating which has significantly facilitated this task. In this paper, ion temperature profiles obtained under electron cyclotron resonance (ECR) and neutral beam injection (NBI) plasma heating conditions are compared with electron temperature and density profiles obtained using a Thomson Scattering diagnostic. From this, similarities in the forms of the ion temperature and electron density profiles are noted for ECR heated plasmas whilst for NBI heated plasmas similarities are observed between ion temperature and electron temperature profiles. Finally, after explaining the different behaviours of the profiles in terms of ion power absorption profile, we demonstrate the possibility of determining ion temperature profiles using two localized ion temperature measurements plus Thomson Scattering profiles.
\end{abstract}

(C) 2010 The Japan Society of Plasma Science and Nuclear Fusion Research

Keywords: ion temperature, Thomson scattering, neutral particle analyzer, TJ-II stellarator

DOI: $10.1585 /$ pfr.5.S2085

\section{Introduction}

The flexible heliac TJ-II is a medium sized stellarator $(R=1.5 \mathrm{~m}, a \leq 0.2 \mathrm{~m}, B<1 \mathrm{~T})$ which can vary its rotational transform profile over a wide range. In TJ-II, the principal ion temperature is routinely measured along a discharge using two Acord-12 neutral particle analyzers (CX-NPA) whose viewing lines-of-sight are symmetric about the plasma centre $(\rho \cong \pm 0.1)$ with $1 \mathrm{~ms}$ of temporal resolution [1]. In addition, a charge exchange recombination spectroscopy (CXRS) system is used to obtain a temperature profile of a fully-stripped impurity ion, covering $\rho=0.2$ to 0.9 , once per plasma discharge [2]. Finally, a Thomson Scattering system provides radial electron temperature and density profiles once per plasma discharge, covering $\rho=-0.7$ to 0.7 [3].

The recent coming on line of two neutral beam injector (NBI) heating systems [4] in TJ-II $\left(\mathrm{H}^{\circ}\right.$, accelerator voltage $\leq 33 \mathrm{keV}$, total injected power $\leq 500 \mathrm{~kW}$ through port) with direct heating of the plasma ions has made the ion channel more important than in previous operational scenario when only electron cyclotron resonance (ECR) heated plasmas were created. The two poloidal viewing CX-NPA on TJ-II permits two ion temperatures to be measured simultaneously at two radial positions separated by a fixed distance. The recent improved reproducibility of

author'se-mail: josepmaria.fontdecaba@ciemat.es
TJ-II discharges due to the new lithium wall coating [5] has enabled us to scan the CX-NPA across the plasma, by moving the line of sight, to obtain ion temperature profiles.

In this work we compare electron temperature and density profiles obtained from the Thomson Scattering diagnostic with majority ion and impurity ion temperature profiles obtained from the CX-NPA and CXRS in order to deduce a general rule for estimating ion temperature profiles when only two local values are known. The estimation of the ion temperature profile will permit more accurate transport studies to be performed.

\section{Experimental Data}

Ion temperature profiles have been obtained with NPA diagnostics [1] for a variety of magnetic configurations, all with average minor radius $\langle a\rangle=0.19 \mathrm{~m}$, with the value of $\iota$ at the edge varying from 1.63 to 1.70 , and the plasma volume varying from 1.079 to $1.098 \mathrm{~m}^{3}$. Table 1 summarizes the main values for the configurations used.

The plasma is created using two gyrotrons tuned to the second harmonic X-mode (53.2 GHz in TJ-II), each with a maximum power of $300 \mathrm{~kW}$ [6]. Once the plasma is stable the NBI is switched on and ECR heating is switched off several milliseconds later, so at the end of a discharge there is pure NBI heated plasma. In the data presented the injector is either co or counter [7], but never both simulta- 
Table 1 The main parameters of the magnetic configurations used in the study. The nomenclature in column 1 represents the central, helical and vertical coil currents. In all cases the plasma averaged minor radius is $0.19 \mathrm{~m}$.

\begin{tabular}{|c|c|c|}
\hline Configuration & $i(\mathrm{a})$ & Plasma volume $\left(\mathrm{m}^{3}\right)$ \\
\hline 1014264 & 1.63 & 1.079 \\
\hline 1004464 & 1.65 & 1.098 \\
\hline 1004665 & 1.67 & 1.096 \\
\hline 1005065 & 1.70 & 1.082 \\
\hline
\end{tabular}

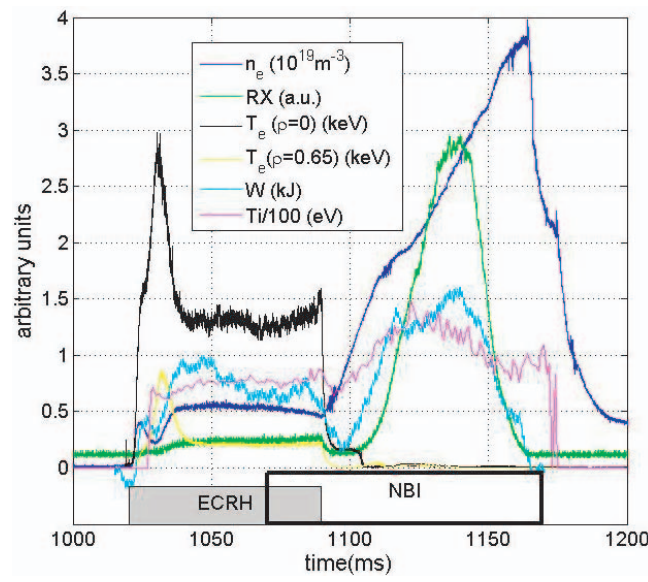

Fig. 1 Heating schemes and main parameters for a typical discharge used for this study. Main plasma parameters represented: line-averaged electron density $\left(n_{\mathrm{e}}\right)$, soft X-ray intensity $(\mathrm{RX})$, edge $(\rho=0.65)$ and central $(\rho=0)$ electron temperature $\left(T_{\mathrm{e}}\right)$, energy content of the plasma $(\mathrm{W})$ and ion temperature $\left(T_{\mathrm{i}}\right)$ at $\rho=0.1$. The switch on periods of ECRH and NBI heating are indicated.

neously. Figure 1 shows the main parameters of a typical NBI discharge used for this study.

Finally, with regard to shapes of profiles from the Thomson Scattering diagnostic, these are classified as dome or bell profiles where bell profiles show a peaked density with rather flat electron temperature, whereas in dome profiles the density is flat and present parabolic temperature profiles [5]. Here, both types of profiles are studied.

\section{Results and Discussion}

In order to compare electron temperature and density profiles with ion temperature profiles, the Thomson Scattering data for each series of shots is combined and fitted using an ad-hoc function (typically, 8 shots in a series). The fitting functions used for the ECR heating phase are:

$$
\begin{aligned}
& T_{\mathrm{e}}(\rho)=T_{\mathrm{e} 0}\left(1-\rho^{\alpha}\right)^{\beta}, \\
& n_{\mathrm{e}}(\rho)=a_{0}+a_{1} \rho+a_{2} \rho^{2}+a_{3} \rho^{3}+a_{4} \rho^{4},
\end{aligned}
$$

while for the NBI heating phase of the discharge the functions are:
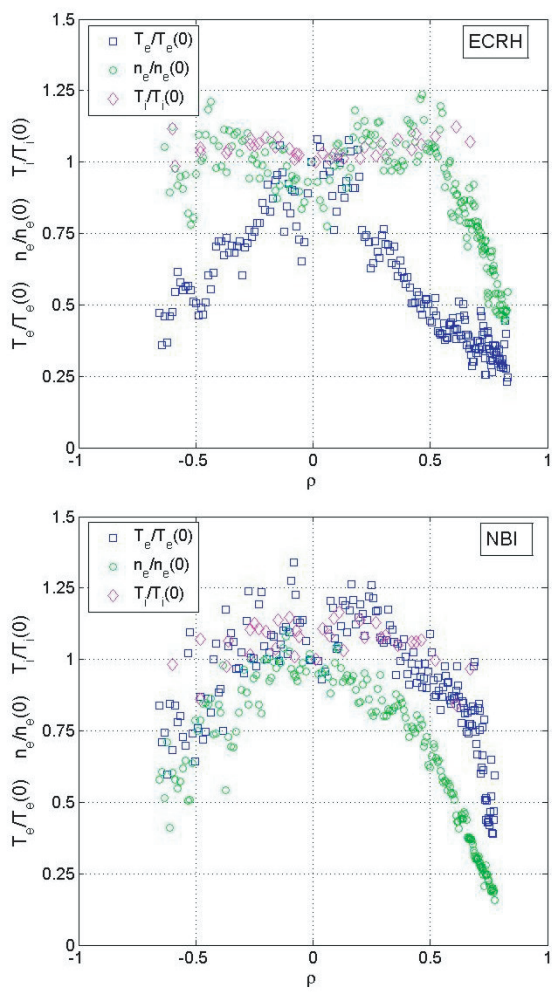

Fig. 2 Relative ion temperature profiles $(\diamond)$ measured by the CX-NPA diagnostics, plus electron temperature ( $\square$ ) and density (O) profiles obtained with Thomson Scattering diagnostic. Each data set is normalized to its central value. Top: ECRH data. Bottom: NBI data.

$$
\begin{aligned}
& T_{\mathrm{e}}(\rho)=a_{0}+a_{1} \rho+a_{2} \rho^{2} \\
& n_{\mathrm{e}}(\rho)=n_{\mathrm{e} 0}\left(1-\rho^{\alpha}\right)^{\beta} .
\end{aligned}
$$

A comparison of the fitting functions with ion temperature profiles is performed by normalizing the fitting functions and the ion temperature profile to their maximums. To evaluate the goodness of the fittings to the ion temperature data the error is computed as the square root of the sum of the differences between the function and experimental points. In NBI discharges, this is consistently smaller for electron temperature profiles than for density ones over the radial range where the data of both diagnostics is reliable, from $\rho=-0.7$ to $\rho=0.7$. Also impurity ion temperature data from the CXRS diagnostic shows better agreement with temperature than density profile shapes near the plasma edge, where this diagnostic has a higher spatial resolution than the CX-NPA.

As seen from Fig. 2, in NBI heated plasmas both the electron and density profile shapes are similar to the ion temperature profile in the plasma centre $(|\rho|<0.3)$. However, the main difference occurs outside the half minornormalized radius, where the electron density falls off suddenly whereas the ion temperature falls less rapidly, like the electron temperature. No significant difference is found between bell or dome profiles, in both cases the most similar profile is the temperature profile. 
For the ECR heating phase, the converse occurs where the ion temperature profile is quite similar to the density profile, (note the highest ion temperature is not in the centre but at $\rho \approx 0.2$ ), where it resembles a typical hollow density profile of ECR plasmas in TJ-II.

The similarity between the ion temperature profile and the electron density, or electron temperature profile depending on the characteristics of the plasma can be explained with respect to the heating of the TJ-II plasmas. There are only two sources that heat ions, collisions with electrons and direct heating by NBI. Ion heating in TJ-II, $\mathrm{Q}_{\mathrm{i}}$, can be estimated using

$$
Q_{\mathrm{i}}=v_{\mathrm{ie}} n_{\mathrm{e}}\left(T_{\mathrm{e}}-T_{\mathrm{i}}\right)+P_{\mathrm{NBI}},
$$

where the first term stands for collisions with electrons and the second one for direct heating through NBI. In the ECH phase only the first term exists. However in the NBI phase this term is also important because in TJ-II, since the critical energy, when starting NBI heating, is about $15 \mathrm{keV}$ for an acceleration voltage of $32 \mathrm{kV}$, the NBI heats mainly the electrons, with only $30 \%$ of the injected power going to the ions [7].

The NBI power absorbed by the ions is modeled, through simulations performed with the FAFNER and EIRENE codes [8], with a function which only depends on line density, total injected power and injector. The heating by collisions with electrons is calculated with the experimental data obtained.

In Fig. 3 it is seen that, during the ECH phase, the profiles of ion temperature and of ion absorbed power by collisions with electrons are very similar, as would be expected given that this is the only heating mechanism. The ion temperature profile is flatter than the heating profile of the ions. This feature can be explained by the size of the ion orbits as explained in Ref. 1.

In the NBI phase ions are heated in two ways, i.e. by collisions with electrons and by direct NBI heating. In Fig. 3, it is seen that the values of both heating mechanisms are similar, and much higher than in ECR heated plasmas. The shape of the collision heating profile in NBI plasmas is nearly parabolic while the direct NBI heating profile resembles a non-centered Gaussian curve. The sum of both curves results in a profile shape similar to that of the ion temperature profile.

The large difference between absorbed power in both types of heating is not followed by a similar increase in the ion temperature. For instance, the central ion temperature in NBI plasmas is about 1.5 times higher than the central ion temperature in ECRH plasmas ( $110 \mathrm{eV}$ compared to $75 \mathrm{eV}$ ), whilst the increase in absorbed power is much higher, about 20 times or more (i.e. $2.5 \cdot 10^{5} \mathrm{~W} \mathrm{~m}^{-3}$ compared to $7.5 \cdot 10^{3} \mathrm{~W} \mathrm{~m}^{-3}$ ). This difference is not surprising because in NBI heated plasmas the density is higher and losses increase because the ion confinement time decreases with power absorbed by ions [9].
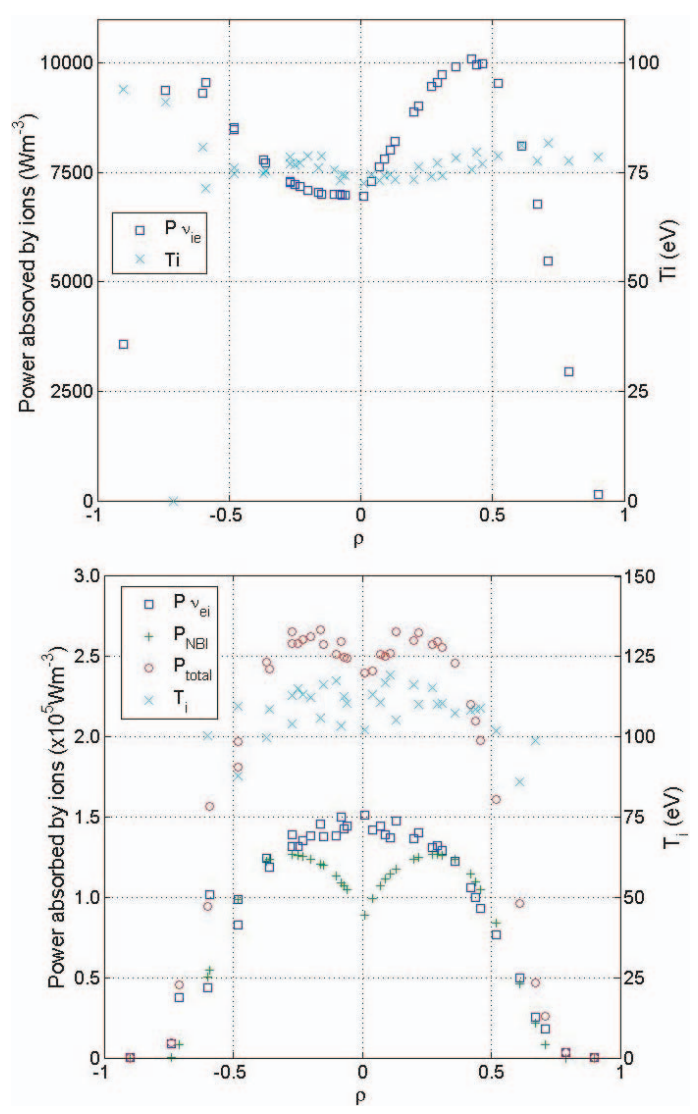

Fig. 3 Power absorbed by plasma majority ions as a function of plasma minor radius. Upper figure: ECR heating, power absorbed by collisions with electrons ( $\square$ ) and ion temperature $(\times)$. Lower figure: NBI heating, power absorbed by ions in collisions with electrons ( $\square$ ), power absorbed by ions from the NBI heating (+), total absorbed power by ions $(\mathrm{O})$, and ion temperature $(\times)$.

\section{Conclusions}

Different series of reproducible discharges have been used to obtain ion temperature profiles. These profiles have been systematically compared with electron temperature and density profiles obtained with the TJ-II Thomson Scattering diagnostic. The results of the comparison show that in ECR heated plasmas the ion temperature and electron density profiles are very similar and conversely in those with NBI heating. This can be explained by the type of heating involved in each situation. This result will permit us to obtain a rough estimation of the ion temperature profile if ion temperature values are available at two points and if the shape of the electron temperature or density profile is available.

Finally, the small increase in ion temperature observed, compared to the large increase in power absorbed by ions, could be explained by a reduction in ion confinement time and by the different densities achieved with ECH and NBI heating schemes. 


\section{Acknowledgements}

J. Arévalo acknowledges financial support from a FPI grant awarded by CIEMAT (BOE resolution $n^{\circ} 171,24-$ 06-2008).

[1] J. M. Fontdecaba et al., Fusion Sci. Tech. 46, 271 (2004).

[2] J. M. Carmona et al., Rev. Sci. Instrum. 77, 10F107 (2006).

[3] J. Herranz et al., Fusion Eng. Des. 65, 525 (2003).

[4] M. Liniers et al., Fusion Technology 1, 307 (1998).

[5] F. L. Tabarés et al., Plasma Phys. Control. Fusion 50, 124051 (2008).
[6] J. M. Fontdecaba et al., Strong Microwaves: Sources and Applications vol 2, 419 (2009).

Edited by A. G. Litvak ISBN: 978-5-8048-0085-8

[7] J. Guasp and M. Liniers, Nuclear Fusion 40, 397 (2000).

[8] J. Guasp et al., Informes Técnicos Ciemat 1050, Ciemat. Madrid. December 2004.

[9] J. M. Fontdecaba et al., "Ion confinement studies in NBI heated TJ-II plasmas using CX-NPA diagnostics" Proceedings 36th EPS Conference on Plasma Physics, June 29 July 3, 2009, Sofia, Bulgaria. 